\title{
Endogenous glucocorticoids and impaired insulin signaling are both required to stimulate muscle wasting under pathophysiological conditions in mice
}

\author{
Zhaoyong Hu, Huiling Wang, In Hee Lee, Jie Du, and William E. Mitch \\ Nephrology Division, Baylor College of Medicine, Houston, Texas, USA.
}

\begin{abstract}
Muscle wasting is associated with a number of pathophysiologic conditions, including metabolic acidosis, diabetes, sepsis, and high angiotensin II levels. Under these conditions, activation of muscle protein degradation requires endogenous glucocorticoids. As the mechanism(s) underlying this dependence on glucocorticoids have not been identified, we analyzed the effects of glucocorticoids on muscle wasting in a mouse model of acute diabetes. Adrenalectomized, acutely diabetic mice given a physiologic dose of glucocorticoids exhibited decreased IRS-1-associated PI3K activity in muscle and progressive muscle atrophy. These responses were related to increased association of PI3K with the glucocorticoid receptor (GR). In mice with muscle-specific GR deletion (referred to as MGRKO mice), acute diabetes minimally suppressed IRS-1-associated PI3K activity in muscle and did not cause muscle atrophy. However, when a physiologic dose of glucocorticoids was given to mice with muscle-specific $I R$ deletion, muscle protein degradation was accelerated. Fluorescence resonance energy transfer and an in vitro competition assay revealed that activated GRs competed for PI3K, reducing its association with IRS-1. Reexpression of WT GRs or those with a mutation in the nuclear localization signal in the muscle of MGRKO mice indicated that competition for PI3K was a prominent mechanism underlying reduced IRS-1-associated PI3K activity. This nongenomic influence of the GR contributes to activation of muscle protein degradation. We therefore conclude that stimulation of muscle proteolysis requires 2 events, increased glucocorticoid levels and impaired insulin signaling.
\end{abstract}

\section{Introduction}

It has been known for decades that endogenous glucocorticoids are required for activation of muscle protein degradation in several models of muscle atrophy (1-5). However, the mechanisms underlying the glucocorticoid requirement have not been identified. We and others have found that stimuli activating muscle wasting are linked to impaired IRS-1-associated PI3K/Akt activity (6-11). For example, decreased IRS-1-associated PI3K/Akt activities can activate the forkhead transcription factor (FoxO) in muscle, leading to transcription of the E3 ubiquitin ligase Atrogin-1/MAFbx $(6,11$, 12). This is relevant because the expression of Atrogin-1/MAFbx is directly related to protein degradation in muscle cells (13). On the other hand, IGF-1 treatment of muscle cells suppresses Atrogin-1 expression and conditional activation of Akt in vivo causes muscle hypertrophy, while overexpression of IGF-1 in muscle blocks angiotensin II-induced muscle atrophy $(5,13,14)$. Thus, changes in IRS-1-associated PI3K in muscle directly influence p-Akt and, ultimately, protein metabolism (15).

Decreased IRS-1-associated PI3K activity in muscle could develop in many catabolic conditions causing accelerated muscle atrophy, including uremia, acidosis, diabetes mellitus, sepsis, or starvation because there also is impaired insulin/IGF-1 signaling $(2,10,16-20)$. Factors other than impaired insulin signaling must be present, however, because mice lacking the insulin receptor in muscle do not develop muscle atrophy (21). Another factor causing

Conflict of interest: The authors have declared that no conflict of interest exists. Citation for this article: J. Clin. Invest. 119:3059-3069 (2009). doi:10.1172/JCI38770. muscle atrophy could be endogenous glucocorticoids. For example, muscle proteolysis in adrenalectomized rodents does not increase even in response to acute diabetes or metabolic acidosis $(1,3)$. In both models, however, it was demonstrated that a physiologic level of glucocorticoids is required to stimulate muscle proteolysis. By itself, however, the same level of glucocorticoids did not stimulate muscle protein degradation. It also has been shown that endogenous glucocorticoids are required for the muscle proteolysis that occurs in models of sepsis or angiotensin II infusion $(4,5)$.

Glucocorticoids have been shown to decrease protein synthesis and stimulate protein degradation in muscle (22). In catabolic conditions, however, the mechanism by which glucocorticoids contribute to muscle wasting is unknown (23). Pharmacological doses of glucocorticoids can cause insulin resistance in rats, leading to decreased PI3K activity in muscle (24). This could occur through genomic and/or nongenomic mechanisms. For example, chronic treatment of cultured muscle cells with dexamethasone (Dex) was shown to increase expression of the p85 subunit of PI3K, and Ueki et al. reported that overexpression of the p 85 subunit in mouse fibroblast cells created an imbalance among p110, p85, and IRS-1, leading to suppression of PI3K activity $(25,26)$. Moreover, Schakman et al. reported that expression of constitutively activated Akt, a dominant negative GSK-3 $\beta$, or a stable $\beta$-catenin can block the muscle atrophy induced by Dex (27). Waddell et al. reported that expression of the E3 ubiquitin ligase, MuRF1, is stimulated by the glucocorticoid receptor (GR) (or FoxO1), leading to increased muscle proteolysis by the ubiquitin-proteasome system (UPS) (28). These reports emphasize the importance of genomic responses to 
A
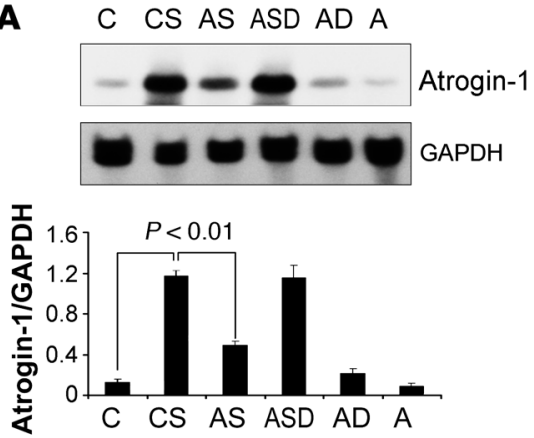

D
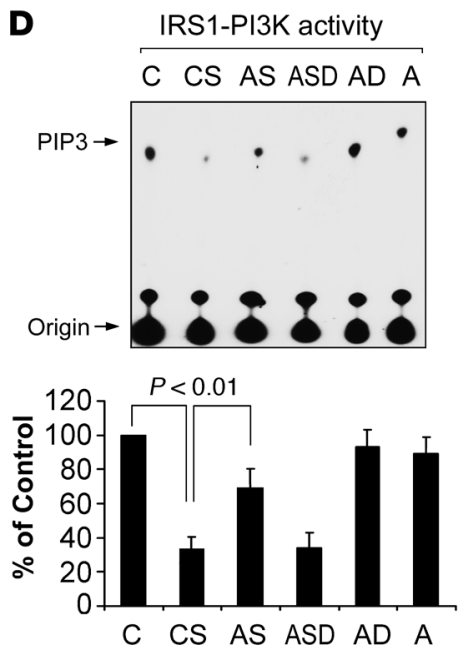

B
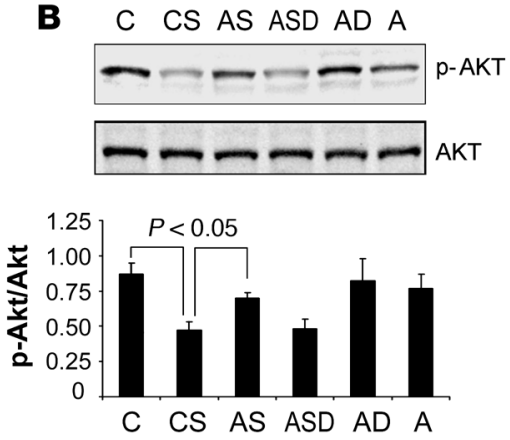

E
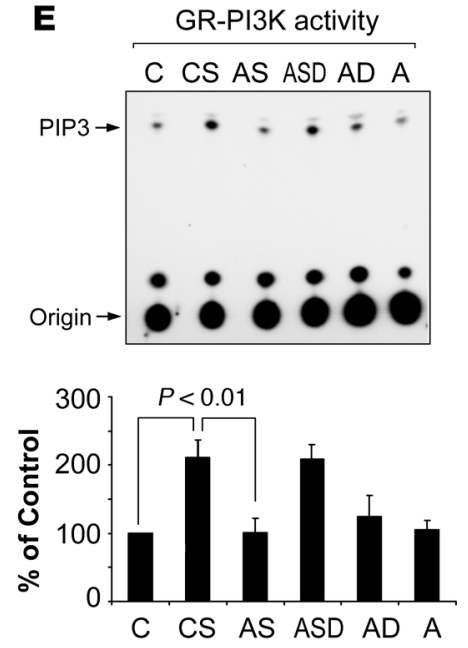

C

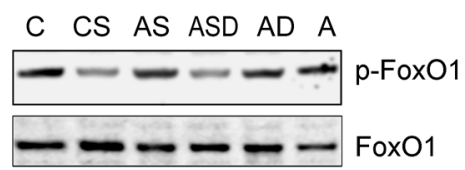

$\mathbf{F}$

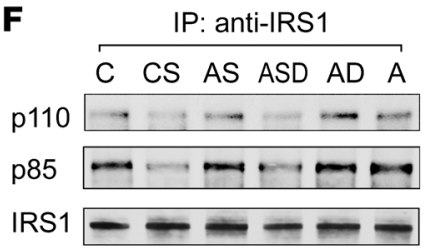

IP: anti-GR

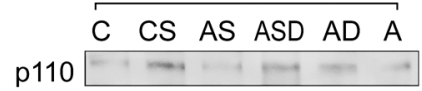

p85

GR

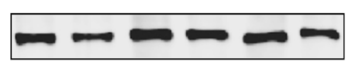

Figure 1

Glucocorticoids are necessary for diabetes-induced activation of proteolytic pathways in muscle. (A) Muscles from control mice (C), diabetic mice (CS), and 4 groups of adrenalectomized mice were examined: AS, diabetes; ASD, diabetes plus a pathophysiologic dose of Dex; AD, with no diabetes but with the same dose of Dex; and A, ADX only ( $n=6$ in each group). Atrogin-1/MAFbx mRNA expression was highest in muscle of CS or ASD mice; it was not increased in AD mice, but was minimally raised in AS mice. (B) In the same groups of mice, the pattern of muscle $p$-Akt levels was reciprocal to that of Atrogin-1/MAFbx mRNA levels. (C) The pattern of p-FoxO1 levels in muscles was parallel to that of $p$-Akt levels. (D) After immunoprecipitation with anti-IRS-1 antibodies, IRS-1-associated PI3K activities in muscles of the different groups paralleled the p-Akt pattern. (E) After immunoprecipitation with anti-GR antibodies, the pattern of GR-associated PI3K activities was reciprocal to that of IRS-1-associated PI3K activities. (F) After immunoprecipitation with anti-IRS-1 or anti-GR antibodies, Western blot analyses of PI3K subunits p110 and p85 revealed the same patterns as in $\mathbf{D}$ and $\mathbf{E}$.

glucocorticoids. But pharmacologic doses of glucocorticoids can also cause rapid, nongenomic responses that influence insulin signaling via membrane-bound, GR-mediated, nongenomic responses or cytosolic GR-mediated, nongenomic effects $(29,30)$. Moreover, receptors of certain steroid hormones including the GR can interact with PI3K to influence its activity $(29,31)$. Whether endogenous glucocorticoids exert metabolic effects on muscle protein metabolism via nongenomic actions has not been established.

We investigated whether a pathophysiologic level of glucocorticoids contributes to muscle atrophy in a catabolic condition, acute diabetes. Using mice with muscle-specific deletion of the GR or muscle-specific deletion of the insulin receptor, we determined that activation of the GR results in an additional suppression of IRS-1-associated PI3K and Akt activities, leading to muscle atrophy. The mechanisms underlying the GR and PI3K interaction involved what we believe to be a new nongenomic action of the activated GR resulting in competition between the GR and IRS-1 for PI3K. This leads to activation of proteolytic pathways and accelerated muscle wasting.

\section{Results}

Evidence that the GR directly interferes with IRS-1-associated PI3K activity in muscle. Previously, we showed that glucocorticoids are required for the increase in muscle protein degradation caused by acute diabetes $(3,19)$. To explore mechanisms by which endogenous glucocorticoids stimulate muscle protein degradation, we investigated responses to acute, streptozotocin-induced (STZ-induced) diabetes in adrenalectomized mice. First, we confirmed that adrenalectomy (ADX) blocks the muscle atrophy induced by acute diabetes (the leftward shift in the distribution of myofiber sizes in diabetic mice was ameliorated by ADX; Supplemental Figure 1A; supplemental material available online with this article; doi:10.1172/ JCI38770DS1). However, when we treated adrenalectomized, diabetic mice with Dex at a dose calculated to mimic the endogenous production of glucocorticoids (1), the leftward shift in myofiber sizes was restored. Second, we evaluated the mRNA of Atrogin-1/MAFbx because there is evidence that it is directly related to the rate of protein degradation in muscle cells (13). There was increased expression of Atrogin-1/MAFbx mRNA (Figure 1A, lanes 
A

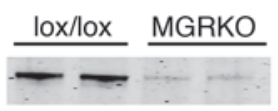

GR
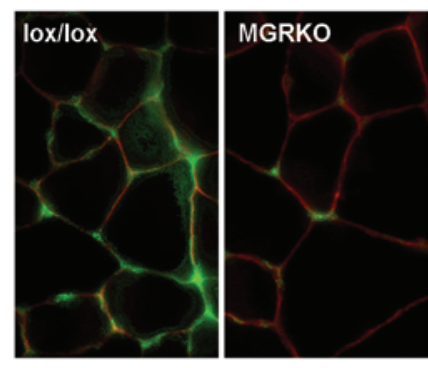

D
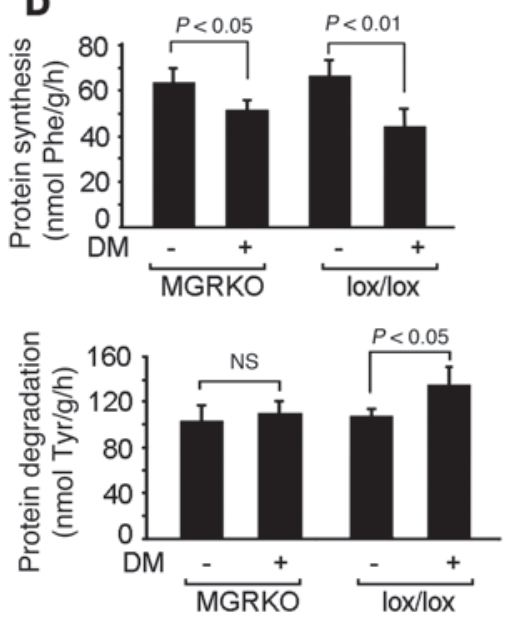
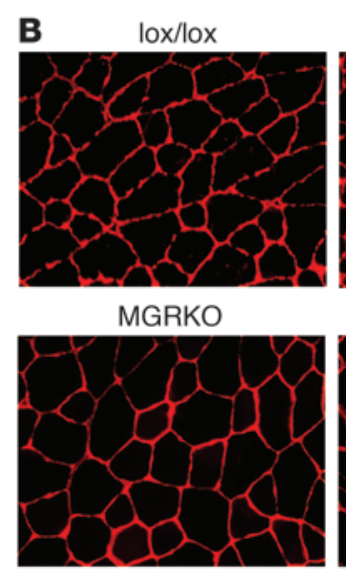

E

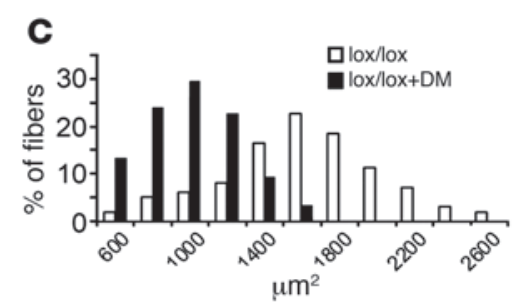

MGRKO+DM
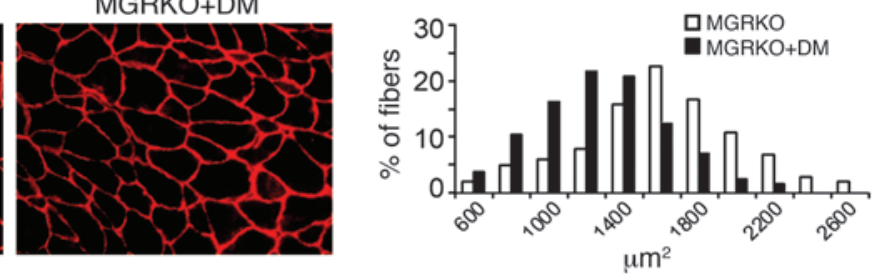
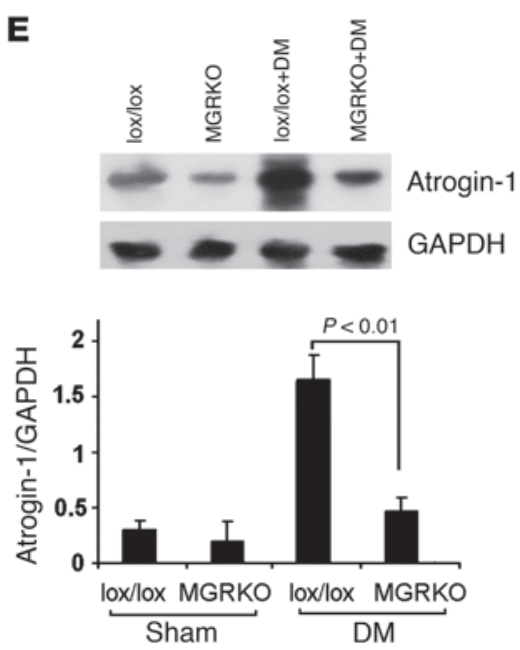

Figure 2

Muscle-specific GR knockout (MGRKO) prevented the accelerated muscle proteolysis induced by diabetes. (A) Immunostaining of dystrophin (red) and GR (green) and Western blotting revealed minimal expression of the GR in myofibers of MGRKO mice. Original magnification, $\times 400$. (B and C) The distribution of muscle fiber sizes in control (Iox/lox) or MGRKO mice with and without acute diabetes (DM) ( $n=3$ in both groups and 300 myofibers examined) differed. The shift of muscle fiber sizes associated with diabetes in lox/lox mice was partially corrected in MGRKO mice. Original magnification, $\times 200$. (D) Rates of protein synthesis and degradation in muscles of MGRKO and lox/lox mice demonstrated that the absence of the GR prevented the increase in protein degradation induced by diabetes ( $n=6$ in each group). Diabetes depressed protein synthesis in muscle of both MGRKO and lox/lox mice. (E) The increased expression of Atrogin-1/MAFbx in muscle of diabetic lox/lox mice was eliminated in muscle of diabetic MGRKO mice ( $n=6$ in both groups).

$\mathrm{C}$ and CS), and ADX largely (but not completely) prevented this response (Figure 1A, lane AS). When glucocorticoids were replaced in the adrenalectomized, diabetic mice, Atrogin-1/MAFbx was stimulated to the same extent as in muscle of acutely diabetic, otherwise intact mice (Figure 1A, lane ASD vs. CS). Note that the same dose of Dex did not stimulate Atrogin-1/MAFbx expression in nondiabetic, adrenalectomized mice (Figure 1A, lane AD). Dex treatment of diabetic, adrenalectomized mice also decreased levels of p-Akt and p-FoxO1, the upstream regulators of Atrogin-1/ MAFbx gene expression (Figure 1, B and C).

Since PI3K activity can regulate muscle atrophy $(6,11,12)$, we measured it in muscle of the different groups of mice. IRS-1associated PI3K was decreased in muscle of diabetic mice or diabetic, adrenalectomized mice treated with Dex. PI3K was minimally decreased in muscle of adrenalectomized diabetic mice (Figure 1D, lane AS). These results suggest that glucocorticoids are required for the downregulation of IRS-1-associated PI3K.
Next, we immunoprecipitated the GR and measured GR-associated PI3K activity. The decrease in IRS-1-associated PI3K in muscle of diabetic, adrenalectomized mice treated with Dex was accompanied by an increase in GR-associated PI3K activity (Figure $1 \mathrm{E})$. The PI3K subunits $\mathrm{p} 85$ and $\mathrm{p} 110$ associated with IRS-1 were decreased in the same fashion as PI3K activity. There also was a reciprocal increase in GR-associated p85 and p110 (Figure 1F). These results indicate that PI3K binding to IRS-1 is inversely related to its binding to the GR.

Muscle-specific GR-knockout mice are resistant to diabetes-induced downregulation of PI3K activity and muscle atrophy. Results from adrenalectomized mice treated with or without Dex suggest that glucocorticoids regulate insulin signaling in a reciprocal fashion. Since ADX can cause stress responses, changes in catecholamine and aldosterone, or secondary changes in pituitary hormones, we investigated the metabolic consequences of endogenous glucocorticoids on muscle wasting by creating muscle-specific GR- 


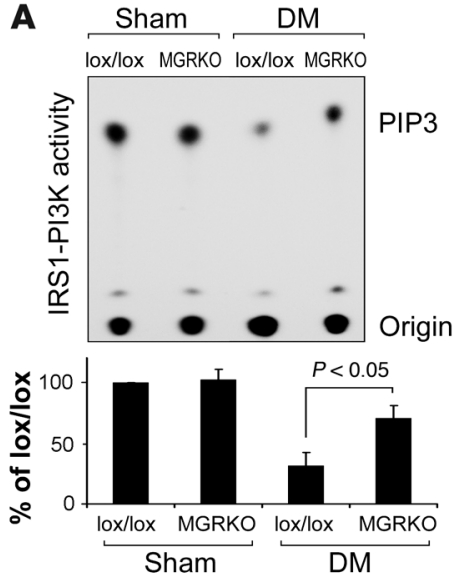

C
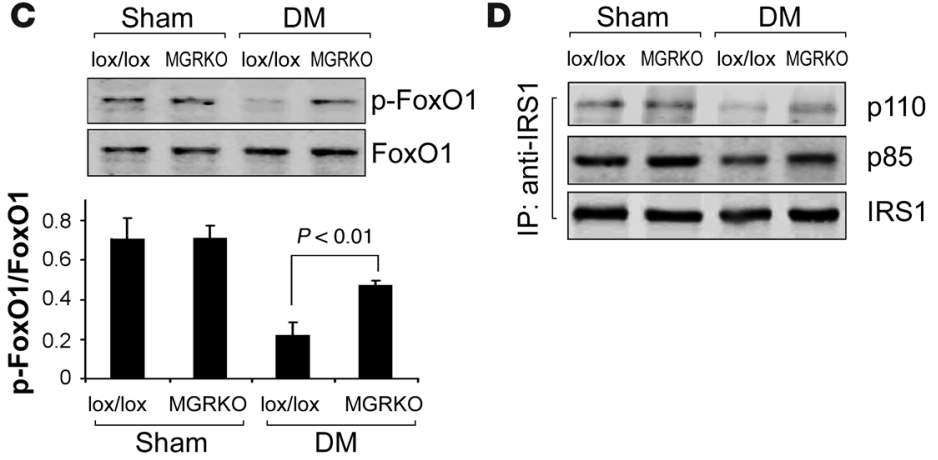

D

B
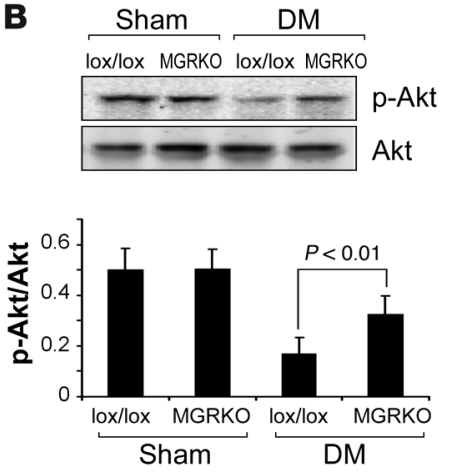
IRS1

\section{Figure 3}

Muscle-specific GR knockout prevented diabetes-induced downregulation of IRS-1-associated PI3K. (A) Suppression of IRS-1-associated PI3K activity in muscle of diabetic lox/lox mice was significantly reversed in muscle of diabetic MGRKO mice ( $n=6$ in each group). (B) Activities of IRS-1-associated PI3K activities (A) are paralleled by levels of p-Akt. (C) IRS-1associated PI3K activities in muscle result in parallel levels of p-FoxO1. (D) The lower levels of IRS-1-associated p110 and p85 subunits of PI3K in muscle of diabetic lox/lox mice are partially corrected in muscle of MGRKO mice.

$(P<0.01)$ in Atrogin-1/MAFbx mRNA and decreases in $\mathrm{p}$-Akt and in $\mathrm{p}$-FoxO1. In MGRKO mice, there was a smaller increase in Atrogin-1/MAFbx mRNA $(P<0.05$; Supplemental Figure 2, B and C).

Glucocorticoids cause muscle atrophy in mice lacking insulin receptors in muscle, a 2-event process. Several reports have documented that endogenous glucocorticoids require another catabolic stimulus to increase muscle protein degradation $(1-3,5)$. Our results demonstrate that the combination of diabetes and glucocorticoids is required to trigger muscle proteolysis. This conclusion must be made with caution, however, because there is potential toxicity from STZ and/ or there could be secondary hormonal responses to low insulin levels. Therefore, we examined mice lacking the insulin receptor, but only in muscle (MIRKO mice). These mice grow normally and have a normal muscle mass (21). We found that MIRKO mice have normal corticosterone production and confirmed that there is a marked reduc-

knockout mice (MGRKO mice). These mice grow normally and have normal myofiber sizes, and using Western blot analysis and immunostaining, we confirmed that the GR is markedly reduced in muscle of MGRKO mice (Figure 2A). After 8 days of diabetes, corticosterone production was significantly increased $(42 \pm 6 \mu \mathrm{g} /$ $\mathrm{kg} / \mathrm{d}$ diabetic, MGRKO vs. $5 \pm 3 \mu \mathrm{g} / \mathrm{kg} / \mathrm{d}$, nondiabetic, lox/lox). In response to diabetes, there was a decrease in the cross-sectional area of myofibers in muscle of control, lox/lox mice but not in muscles of MGRKO mice (Figure 2, B and C).

To explore why muscle atrophy did not occur in diabetic MGRKO mice, we measured protein synthesis and degradation in extensor digitorum longus (EDL) muscles. Diabetes significantly decreased protein synthesis in muscles of both lox/lox and MGRKO mice (Figure 2D). Diabetes also stimulated protein degradation, but only in muscles of lox/lox mice. In muscles of lox/lox mice, diabetes also induced Atrogin-1/MAFbx expression (Figure 2E). Again, this response was absent in muscle of MGRKO mice.

In muscles of acutely diabetic lox/lox mice (as in adrenalectomized mice), IRS-1-associated PI3K activity and IRS-1-associated p85/ p110 levels were suppressed to a greater extent than those in muscles of diabetic, MGRKO mice (Figure 3, A and B). Changes in p-Akt and p-FoxO1 levels followed a similar pattern (Figure 3, C and D).

Eliminating the GR from muscle also benefited another condition associated with decreased IRS-1-associated PI3K activities in muscle, namely, fasting. After 36 hours of food deprivation, plasma corticosterone levels in the MGRKO and control lox/lox mice were 4 -fold higher than those in nonfasting mice $(P<0.01)$. Food deprivation led to a leftward shift in the distribution of myofibers in lox/lox mice and minimally in muscle of MGRKO mice (Supplemental Figure 2A). In muscle of lox/lox mice, there was an increase tion in insulin receptors in the muscles (Supplemental Figure 3). To test for the absence of insulin receptors in muscle, we injected MIRKO and lox/lox mice with insulin. As shown in Figure 4A, insulin-stimulated, IRS-1-associated PI3K activity was markedly lower in muscles of MIRKO versus lox/lox mice.

MIRKO and lox/lox mice were treated with the physiologic dose of glucocorticoids, and 8 days later, we measured muscle protein synthesis and degradation. Dex administration did not suppress muscle protein synthesis in either mouse strain (Figure 4B). However, it significantly increased muscle protein breakdown in MIRKO but not in lox/lox, control mice (Figure 4C). Muscle atrophy in Dex-treated MIRKO mice was detected by the leftward shift in the distribution of cross-sectional areas of myofibers (Figure 4, $\mathrm{D}$ and $\mathrm{E})$. There also was an increase in Atrogin-1/MAFbx expression (Figure 4F), along with a decrease in p-Akt and p-FoxO1 (Figure 5, A and B). Thus, a physiologic dose of glucocorticoids causes (i.e., muscles of MIRKO mice).

We measured IRS-1-associated PI3K activity in muscle of MIRKO mice treated with Dex and found it was reduced (Figure 5C). In contrast, Dex did not suppress IRS-1-associated PI3K in muscle of control lox/lox mice. Next we examined whether glucocorticoids would affect the distribution of PI3K between IRS-1 and GR in muscle of MIRKO mice. As shown in Figure 5D, GR-associated PI3K increased in muscle of Dex-treated MIRKO mice; this did not occur in muscles of lox/lox mice. Dex also increased GR-associated p110 and p85 subunits of PI3K while decreasing IRS-1-associated p110 and p85 subunits in muscle of MIRKO mice (Figure 5, E and F).

To test whether similar events occur with food deprivation, which decreases insulin signaling, we measured plasma corticosterone in muscle atrophy only when insulin signaling in muscle is defective 

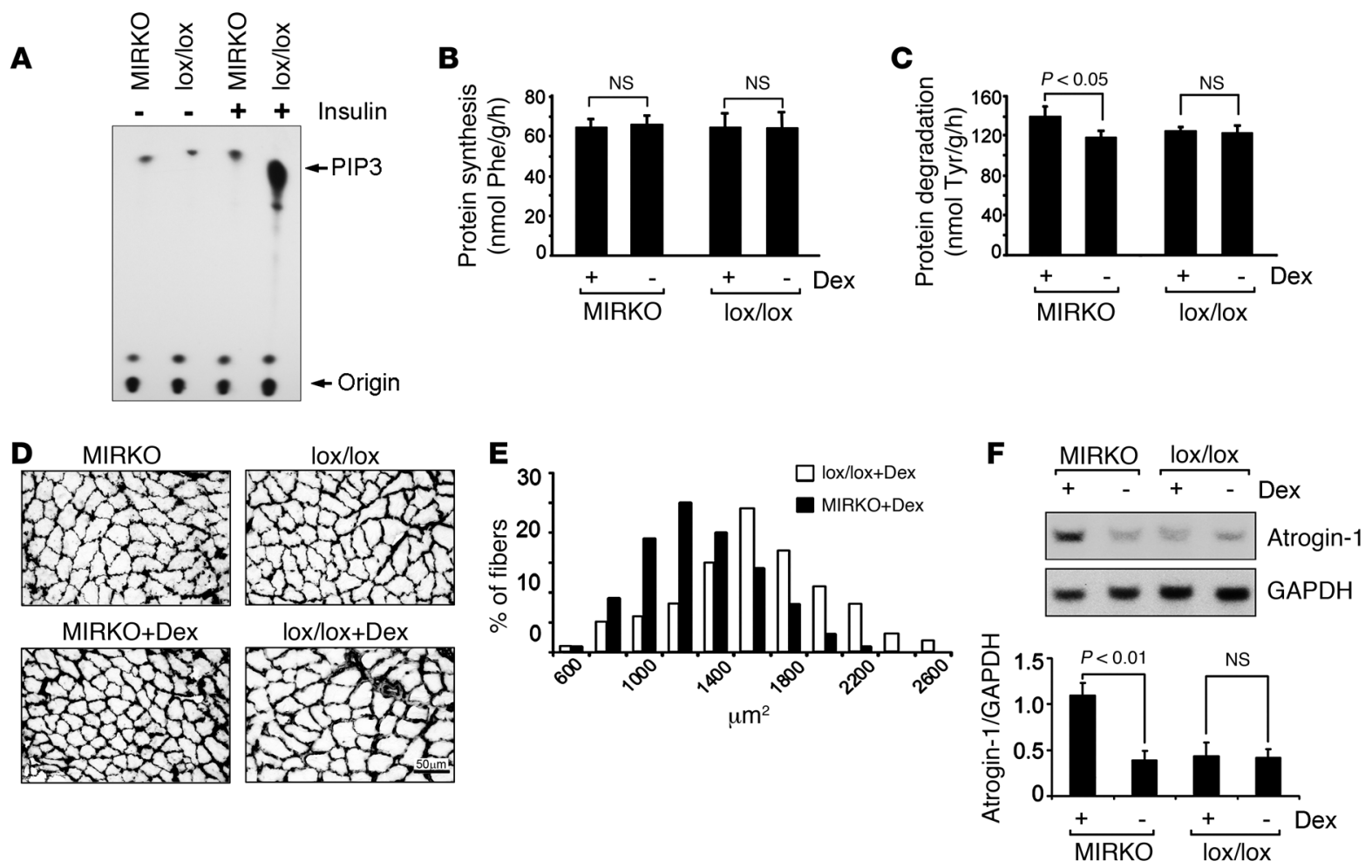

\section{Figure 4}

Both abnormal insulin signaling and increased glucocorticoid production are required to induce muscle atrophy. (A) Muscle-specific knockout of the insulin receptor in mice (MIRKO) prevented the sharp increase in IRS-1-associated PI3K activity stimulated by insulin (representative blot from 3 separate experiments). PIP3, phosphatidylinositol. (B and C) A high physiologic dose of glucocorticoids did not change protein synthesis but stimulated protein degradation in muscle of MIRKO mice ( $n=8$ in each group). (D and E) MIRKO mice treated with Dex developed muscle atrophy, as indicated by a shift to smaller sizes of myofibers. Scale bar: $50 \mu \mathrm{m}$. (F) MIRKO mice treated with Dex exhibited increased expression of Atrogin-1/MAFbx in muscle ( $n=3$ in each group).

lox/lox and MIRKO mice. After 36 hours of food deprivation, plasma corticosterone was approximately 6-fold higher in MIRKO and lox/lox mice compared with nonfasted mice $(P<0.01)$. In lox/lox and MIRKO mice, food deprivation caused a marked leftward shift in the distribution of cross-sectional areas of myofibers (Supplemental Figure $4 \mathrm{~A}$ ). In muscle of both groups of mice, there was a significant $(P<0.05)$ increase in Atrogin-1/MAFbx mRNA and a decrease in p-Akt and p-FoxO1 (Supplemental Figure 4, B and C). Thus, the 2 events - the inability of muscles of MIRKO mice to respond to insulin plus activation of the GR - result in muscle atrophy.

Identification that the GR-induced muscle atrophy involves a nongenomic action of glucocorticoids. Glucocorticoids can induce transcriptional and transactivation/repression responses as well as nongenomic responses (30, 32-34). To determine whether GR-induced muscle atrophy involves genomic responses, we mutated the nuclear location signal sequence (NLSS) from Lys to Asn at site 515 (35). As shown in Figure 6A, the mutated GR did not enter the nucleus of COS1 cells when Dex was added. Next, we used electroporation to transfer the wild-type or mutant GR into myofibers of MGRKO mice; these mice were then treated to produce acute diabetes. In muscles of acutely diabetic MGRKO mice, introduction of either the wild-type or the mutant GR caused a decrease in the sizes of myofibers compared with results from nondiabetic MGRKO mice (Figure 6B). We note that the myofiber sizes of diabetic MGRKO mice reexpressing the mutated GR were larger than myofibers expressing the wild-type GR. The expression of Atrogin-1/MAFbx in muscles of nondiabetic, MGRKO mice was minimal (Figure 6C). But in muscles of diabetic MGRKO mice, expression of Atrogin-1/ MAFbx increased more in muscles reexpressing the wild-type GR compared with those reexpressing the mutant GR (Figure 6C). Thus, the major influence of the activated GR on stimulating muscle atrophy involves nongenomic mechanisms. Besides the nongenomic effect of the GR, however, there may be a genomic effect, because the expression of Atrogin-1/MAFbx was lower in response to reexpression of the mutated GR.

Activated GR competes with IRS-1 for PI3K. Based on immunoprecipitation experiments, the GR can physically associate with PI3K. To analyze this association, we cotransfected COS1 cells with both the GR-cyan fluorescent protein (GR-CFP) and the p85-yellow fluorescent protein (p85-YFP) and measured fluorescence resonance energy transfer (FRET) (36). Treatment with 50 nM Dex produced a time-dependent energy transfer from CFP to YFP within minutes, demonstrating the physical association of the 2 proteins (Figure 6A and Supplemental Figure $2 \mathrm{C}$ ). Even though Dex led to nuclear accumulation of the GR, a large fraction of GR remained in the cytosol, accounting for the physical interaction between GR and p85 in the cytosol (Figure 6B).

To demonstrate that the activated GR can compete with IRS- 1 for $\mathrm{PI} 3 \mathrm{~K}$, we used an in vitro assay based on adding recombinant GR to determine whether it would reduce the PI3K that is associated with 

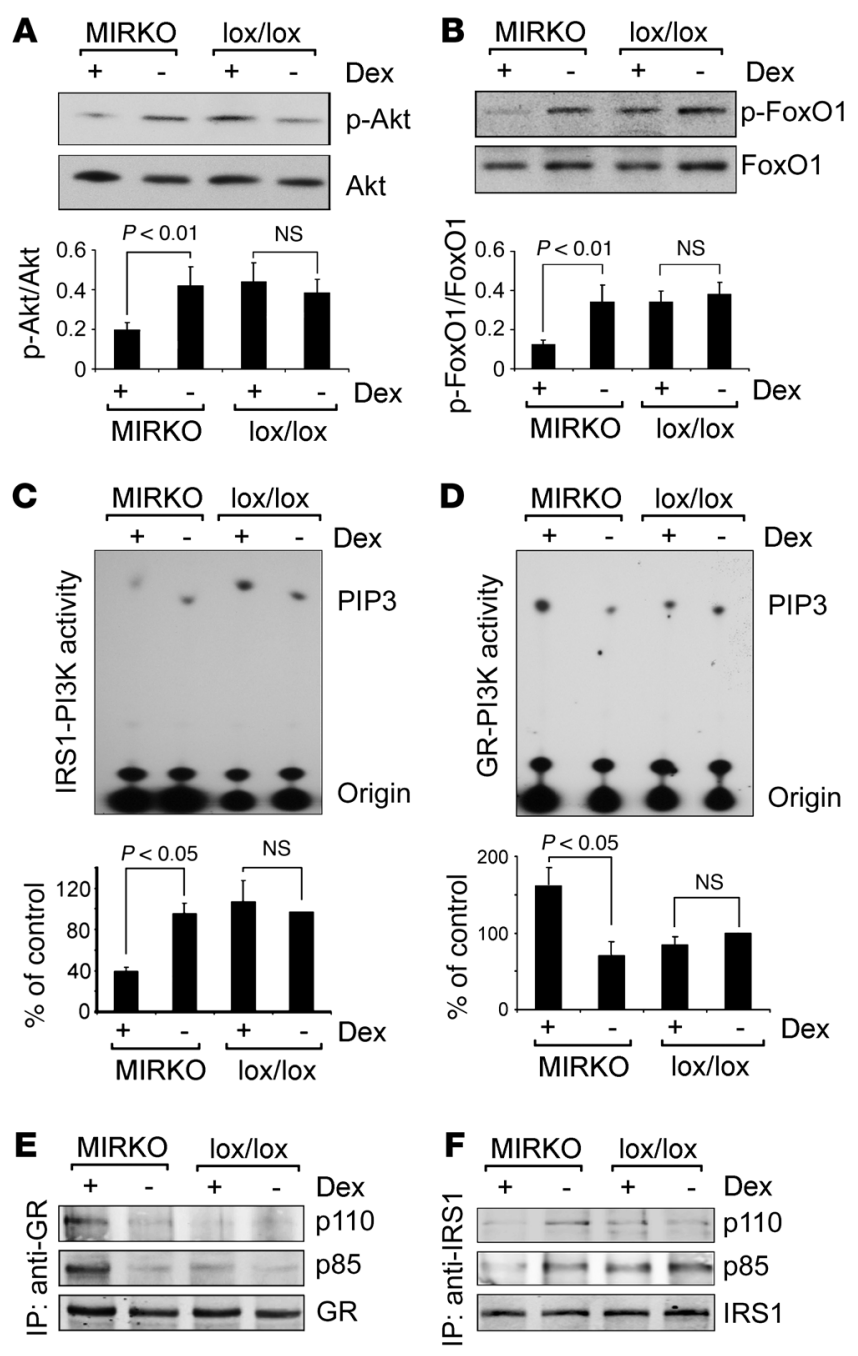

IRS-1. As shown in Figure 6C, increasing the concentration of recombinant GR in cell lysates from insulin-treated C2C12 myotubes led to a progressive decrease in IRS-1-associated PI3K activity when Dex was present. Likewise, adding recombinant GR decreased the binding of the PI3K subunit proteins p110 and p85 to IRS-1 (Figure 6C).

The decrease in IRS-1-associated PI3K activity in muscle of MIRKO but not wild-type mice suggests that insulin might overcome the influence of glucocorticoid-induced changes in IRS-1associated PI3K activity. To test this possibility, we treated C2C12 myotubes with Dex for 30 minutes and then added insulin. As shown in Figure 7D, GR activation alone increased the PI3K activity associated with GR. GR activation also decreased IRS-1-associated PI3K activity (Figure 7D). These changes in PI3K activity were closely linked to binding of the PI3K subunits (p110 and p85) to either the GR or IRS-1 (Figure 7D). Notably, the glucocorticoidinduced decrease in IRS-1-associated PI3K activity was eliminated by addition of insulin. This result is consistent with our finding that insulin rapidly blocks the excessive muscle proteolysis induced by acute diabetes (3).

\section{Discussion}

There are differences in the responses to physiologic and pharmacologic doses of glucocorticoids in terms of stimulating muscle

\section{Figure 5}

The increase in muscle proteolysis in MIRKO mice induced by a high physiologic dose of glucocorticoids is due to suppression of IRS-1associated PI3K/Akt. (A) Dex suppressed p-Akt levels, but only in muscle of MIRKO mice ( $n=6$ in each group of MIRKO and control mice). (B) The increase in p-Akt was associated with a significant decrease in p-FoxO1. (C and D) Dex decreased IRS-1-associated PI3K activity in muscle of MIRKO mice and increased GR-associated PI3K activity. ( $E$ and $\mathbf{F}$ ) The changes in $\mathrm{PI}$ KK activity were paralleled by changes in PI3K subunit proteins $\mathrm{p} 110$ and p85 associated with IRS-1 or the GR.

atrophy. Catabolic conditions such as diabetes, acidosis, sepsis, uremia, fasting, and excess angiotensin II require physiologic levels of glucocorticoids to activate muscle proteolysis in vivo. Our results indicate that in such catabolic conditions, endogenous glucocorticoids activate the GR, which competes with IRS-1 for PI3K, further reducing IRS-1-associated PI3K activity. The lower IRS-1-associated PI3K level reduces p-Akt, thus activating proteolytic mechanisms producing muscle atrophy. Although pharmacologic doses of glucocorticoids are also associated with decreased PI3K activity and activation of the UPS, it is unclear whether this is the sole reason for their ability to stimulate muscle atrophy (23). Other mechanisms induced by pharmacologic doses of glucocorticoids can involve nonphysiologic responses, such as stimulation of membrane-bound or cytosolic, GR-mediated, nontranscriptional responses $(29,30)$. The role of these pathways in activating muscle protein metabolism is still controversial (23).

The present experiments were directed at determining how endogenous glucocorticoids contribute to the muscle wasting that occurs in catabolic conditions characterized by defects in insulin signaling in muscle. Specifically, we uncovered metabolic mechanisms by which endogenous glucocorticoids cause a further reduction in IRS-1-associated PI3K and p-Akt activities. Evidence for a required influence of both decreased IRS-1-associated PI3K and increased glucocorticoid production was obtained in MIRKO mice. Despite having impaired insulin signaling in muscle, these mice do not have accelerated muscle proteolysis. But when they are given a physiologic level of glucocorticoids equivalent to the level induced in stressed rodents (1), muscle protein degradation increased. Thus, in certain catabolic conditions, a 2-event process, impaired insulin signaling and increased glucocorticoid production, acts to downregulate IRS-1-associated PI3K activity further, leading to an increase in muscle protein degradation.

The muscle loss in models of diabetes is blocked by ADX, suggesting that glucocorticoids are necessary for stimulating muscle proteolysis $(3,6,19)$. But ADX or the presence of diabetes can change adrenal hormone levels and activate secondary responses (e.g., changes in growth hormone, glucagon, or cytokines) that can change muscle protein metabolism. To avoid these problems, we studied MGRKO (GR deficiency in muscle only) and MIRKO mice (insulin deficiency in muscle only). Both strains of mice grow normally and have normal levels of PI3K in muscle and normal glucocorticoid production. But MGRKO mice are resistant to the accelerated muscle proteolysis induced by diabetes or fasting despite an increase in glucocorticoids (Figure 2 and Supplemental Figure 2). In contrast, when we treated MIRKO mice with a physiologic dose of glucocorticoids, there was activation of muscle proteolysis (Figure $4, C$ and D). Likewise, fasted MIRKO mice had an increase in plasma glucocorticoids and muscle wasting (Supplemental Figure 4). These results demonstrate for the first time to our knowledge that 
A

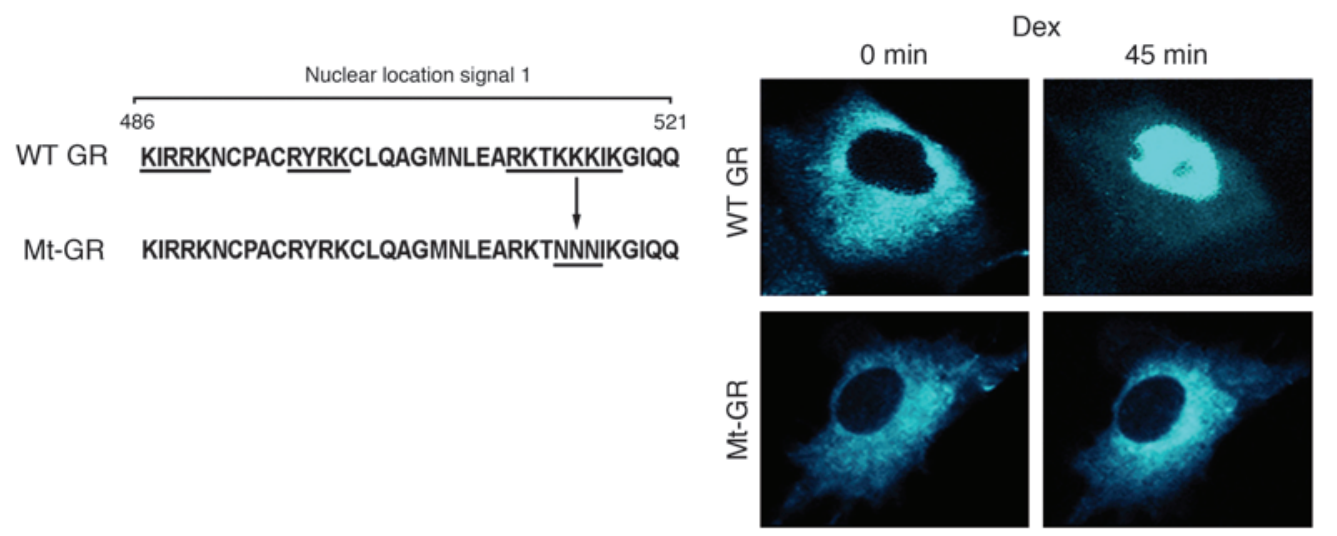

B

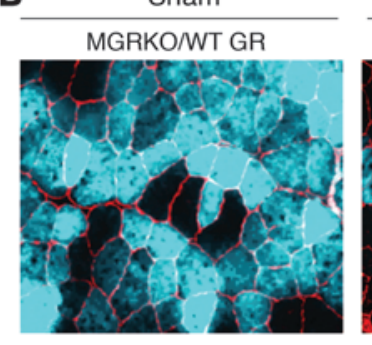

C

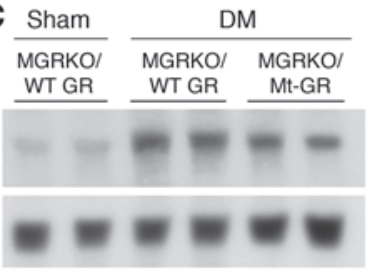

DM
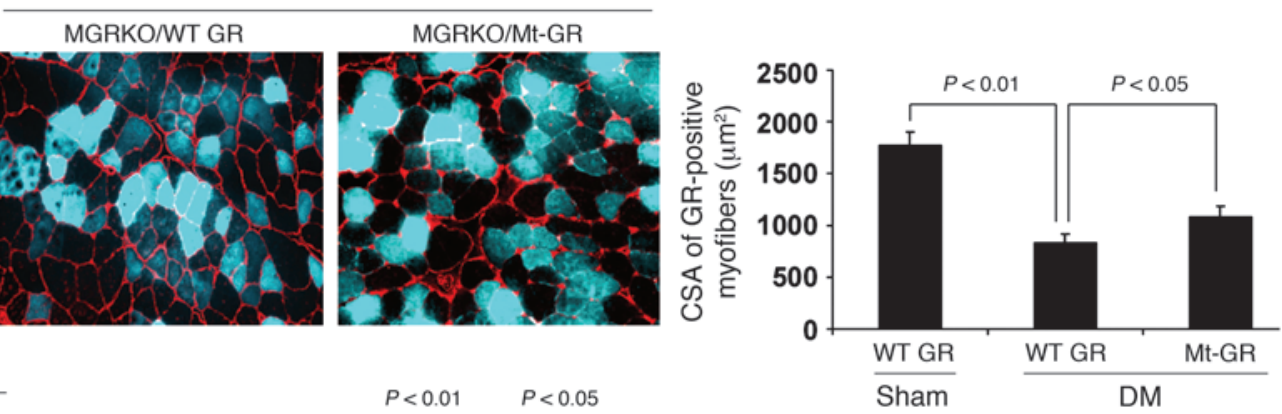

Atrogin-1

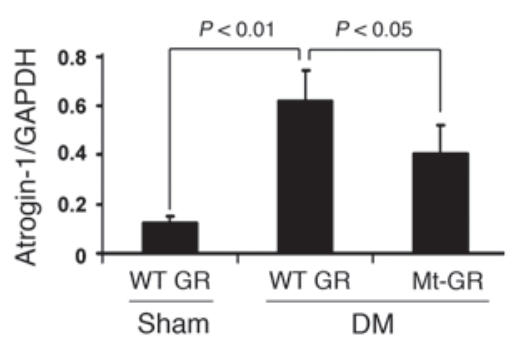

GAPDH

\section{Figure 6}

Identification of a nongenomic effect of the GR on muscle atrophy induced by diabetes. (A) Left panel shows the location of the mutation that blocks the NLS1 of the GR. The right panel shows that the mutated GR (Mt-GR) does not translocate to the nucleus after addition of Dex. Original magnification, $\times 200$. (B) Blue-green indicates electroporation-induced expression of wild-type or mutated GR in myofibers of TA muscles of MGRKO mice ( $n=5$ in each group). Compared with nondiabetic MGRKO mice $(n=3$ mice), acute diabetes decreased the average size (cross-sectional area [CSA]) of myofibers reexpressing the wild-type or mutant GR. The graph shows that the wild-type GR induced a more severe atrophy compared with the mutated GR. Original magnification, $\times 200$. (C) Muscles from MGRKO mice treated as in B were examined for Atrogin-1/MAFbx mRNA. The graph shows that reexpression of the wild-type GR raised mRNA levels more than the mutant GR.

a combination of deficient insulin signaling and activation of the GR in muscle are required to decrease IRS-1-associated PI3K, leading to accelerated muscle protein loss (Figure 5). Consequently, our investigations of MGRKO and MIRKO mice have provided insights into the effects of the interaction between GR and insulin signaling on muscle protein metabolism in vivo.

We found that when glucocorticoid production was eliminated by ADX, diabetes minimally reduced IRS-1-associated PI3K (Figure 1). But replacement with a physiologic dose of glucocorticoids sharply decreased IRS-1-associated PI3K in muscle of these mice (Figure 1D). This response led to a decrease in myofiber sizes (Supplemental Figure 1A), which was related to activation of proteolytic pathways and an increase in protein degradation (Supplemental Figure 1). Interestingly, ADX alone did not completely block the reduction in fiber sizes, perhaps because the adrenalectomized mice still exhibited a decrease in protein synthesis (Supplemental Figure 1B).
How do impaired insulin action and glucocorticoids cause muscle atrophy in catabolic conditions? A traditional explanation is that impaired insulin signaling downregulates PI3K activity, triggering proteolytic pathways (15). But impaired insulin signaling by itself does not cause muscle atrophy. We found that the activated GR competes with IRS-1 for PI3K, leading to lower $\mathrm{p}$-Akt and stimulation of proteolytic pathways. Evidence for this additional mechanism includes our FRET analysis, which demonstrated an intracellular association between GR and p85 (Figure 7). The association was observed within minutes of adding glucocorticoids, and it increased with time. Second, addition of activated GR to immunoprecipitated IRS-1-associated PI3K decreased the activity of IRS-1-associated PI3K and the binding of PI3K subunits to IRS-1 (Figure 7C). Since PI3K activation of Akt occurs as a membrane-based event, GR activation could sequester PI3K away from the membrane to decrease p-Akt (37). 
A
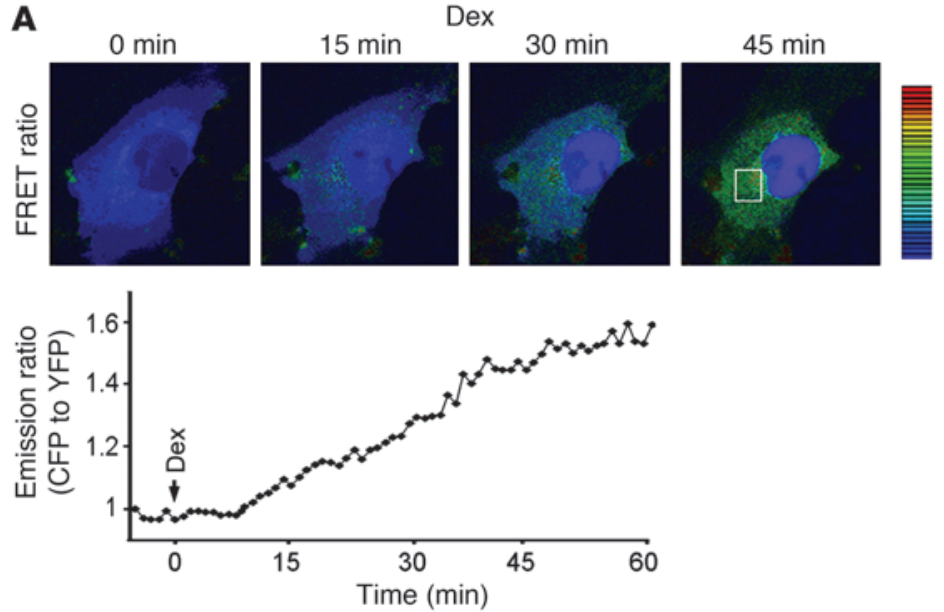

B

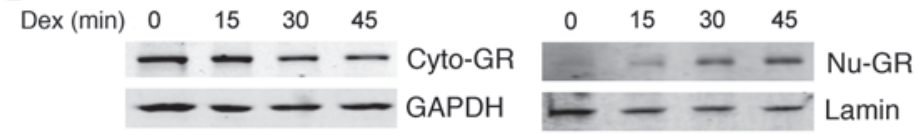

C
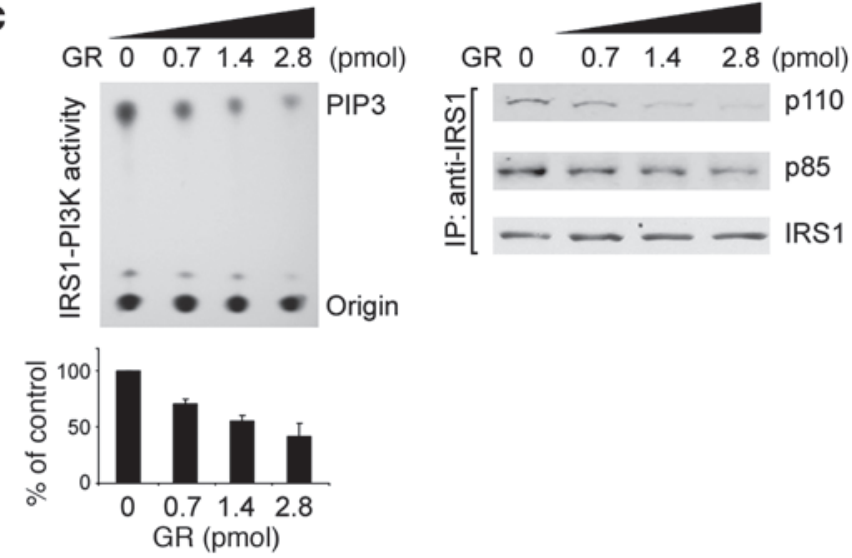

D
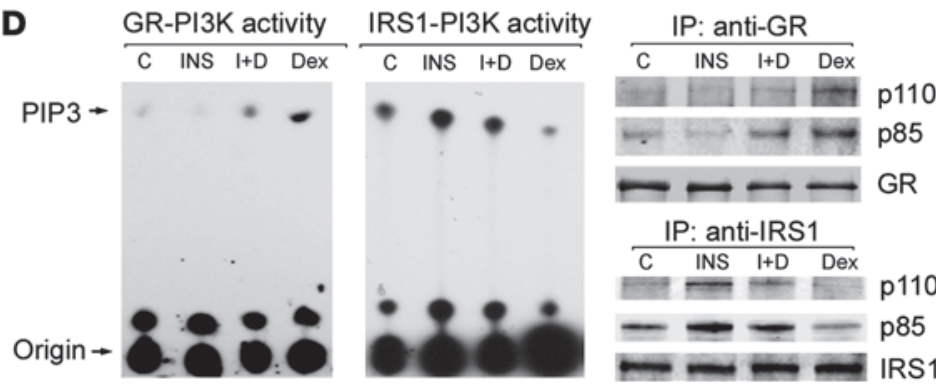

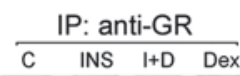

\section{Figure 7}

The activated GR competes with IRS-1 for PI3K. (A) Dex treatment of COS1 cells transfected with GR-CFP (donor) and p85-YFP (recipient) stimulated a time-dependent interaction between GR and p85, as assessed by FRET analysis ( $n=3$ separate experiments). The box shows the area where emission ratio was measured. (B) After addition of Dex, a substantial portion of the GR was in the cytosol (Cyto) despite accumulation of the GR in the nucleus $(\mathrm{Nu})$. (C) In an in vitro competition assay, addition of increasing amounts of recombinant GR led to a progressive decrease in the PI3K activity and PI3K subunits associated with IRS-1 $(n=3)$. (D) C2C12 muscle cells were treated with insulin (INS), insulin plus Dex $(\mathrm{I}+\mathrm{D})$, or Dex alone. Dex alone increased GR-associated $\mathrm{PI} 3 \mathrm{~K}$ as well as PI3K subunit proteins. Cells treated with insulin alone had increased IRS-1-associated PI3K activity and minimal GR-associated PI3K activity. Treatment with insulin largely overcame the Dex-induced depression of IRS-1-associated PI3K activity.

nucleus. In diabetic MGRKO mice, myofibers reexpressing either the wild-type or mutated GR exhibited atrophy (Figure 6B). For 2 reasons, these results cannot exclude contributions of genomic influences of GR on muscle atrophy and Atrogin-1/MAFbx expression. First, the myofiber sizes associated with introducing the wild-type GR in muscles were smaller than those in muscles expressing the mutated GR (Figure 6). This might represent a decrease in protein synthesis (Figure 1D) or an unidentified genomic response. Second, the Atrogin-1/MAFbx level was significantly greater in muscles expressing the wild-type GR compared with muscle expressing the mutant GR and hence could reflect transactivation of Atrogin-1/MAFbx expression because the promoter region of Atrogin-1/MAFbx does not contain a glucocorticoid response element $(11,23)$.

Regarding the specificity of the competition between the GR and IRS-1 for PI3K, we found that giving Dex to normal or adrenalectomized mice as well as treating cultured myotubes with Dex did not increase IRS-1 Ser307 phosphorylation (data not shown), nor did Dex change tyrosine phosphorylation of the insulin receptor or IRS-1 in muscle cells (Supplemental Figure 1C).

Why does a physiologic glucocorticoid dose by itself fail to initiate protein degradation? When myotubes were treated with Dex, addition of insulin sharply reduced the increase in GR-associated PI3K activity; it also raised IRS-1associated PI3K activity (Figure 7D). This result is consistent with a 2-event process in catabolic conditions (e.g., uremia, metabolic acidosis, sepsis, and excess angiotensin II),

Indeed, the FRET results suggest that following activation of the GR, there is relocation of PI3K away from the membrane (Figure $7 \mathrm{~A})$. Another potential explanation is that the activated GR binds to and suppresses the kinase activity of PI3K, since the GR can bind to JNK, decreasing its kinase activity (38). The consequence of decreased PI3K activity is lower p-Akt, which stimulates Atrogin-1/MAFbx expression and muscle protein degradation (15). Thus, the combination of GR activation and impaired insulin signaling initiates muscle atrophy (Figure 1).

These glucocorticoid-induced changes suggest there is a nongenomic response to the activated GR (30). We examined this possibility by mutating the GR to prevent it from translocating to the since these conditions not only impair insulin signaling and raise glucocorticoid production, but they also cause muscle atrophy. Moreover, when MIRKO mice were treated with Dex, there was decreased IRS-1-associated PI3K and muscle atrophy. In contrast, Dex administration to lox/lox mice with intact insulin signaling in muscle had minimal changes in IRS-1-associated PI3K activity (Figure 5). These results offer an explanation for why endogenous glucocorticoids alone do not stimulate muscle protein degradation in vivo, because a rise in glucocorticoids will increase insulin to overcome proteolytic responses to glucocorticoids. But with fasting, we presume that suppression of insulin secretion persists despite an increase in glucocorticoids, because p-Akt is reduced in 


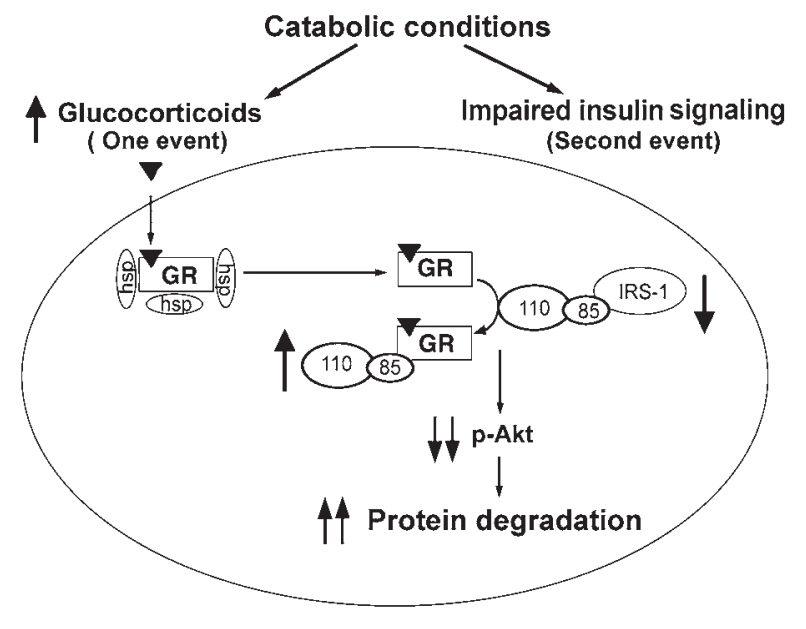

Figure 8

Catabolic conditions not only impair insulin signaling in muscle (one event) but also stimulate glucocorticoid production (second event), leading to muscle atrophy. Glucocorticoids activate the GR in muscle, leading to a competition with IRS-1 for association of PI3K subunits p110 and p85. The association of these subunits with the GR reduces IRS-1-associated PI3K activity and p-Akt. These changes stimulate protein degradation pathways, leading to muscle atrophy.

muscle (Supplemental Figure 2C and Supplemental Figure 4C). The decrease in p-Akt leads to muscle atrophy (15).

In summary, our results demonstrate that the activated GR competes with IRS-1 for PI3K activity, as shown in Figure 8. We conclude that 2 events, impaired insulin signaling accompanied by an increase in endogenous glucocorticoids, combine to stimulate muscle wasting in catabolic condition such as acute diabetes, type 2 diabetes, metabolic acidosis, angiotensin II infusion, sepsis, or starvation.

\section{Methods}

Antibodies. Antibodies against phospho-Akt (Ser473), phospho-FoxO1 (Ser256), and PI3K (p110 $\alpha$ ) were purchased from Cell Signaling Technology. Antibodies against Akt, FoxO1, and the GR were from Santa Cruz Biotechnology Inc. Anti-IRS-1, anti-insulin receptor $\beta$, and anti-Cre recombinase antibodies were from Millipore.

Animal model. The experiments were approved by the Baylor College of Medicine Animal Care Committee. Mice bearing MCK-Cre or with loxP floxed insulin receptor were provided by C.R. Kahn (Joslin Diabetes Center, Boston, Massachusetts, USA); they were used to generate MIRKO mice (21). Mice with loxP floxed GR were provided by L. Muglia (Washington University, St. Louis, Missouri, USA) and used to generate MGRKO mice. For glucocorticoid responses, MIRKO and control (lox/lox) mice were injected with Dex $(20 \mu \mathrm{g} / \mathrm{kg} / \mathrm{d})$ for 8 days. This dose was calculated based on the endogenous secretion rate present in stressed rodents as described by Slusher and Roberts and does not cause muscle protein abnormalities in adrenalectomized rodents $(1,39)$. Gastrocnemius muscles were removed from anesthetized mice, immediately frozen in liquid nitrogen, and stored at $-80^{\circ} \mathrm{C}$. Tibialis anterior (TA) muscle and bone were removed and fixed in $10 \%$ formalin; the muscles were removed and imbedded in HistoPrep medium (Fisher). Mixed fiber, plantaris muscles were used to measure protein synthesis and degradation as described (1).

To induce diabetes, we injected 12 -week-old C57BL/6 mice (The Jackson Laboratory) or MGRKO mice intraperitoneally with 2 doses of $150 \mathrm{mg} /$ $\mathrm{kg} / \mathrm{d} \mathrm{STZ}$ in $0.1 \mathrm{M}$ citrate buffer ( $\mathrm{pH} 4$ ); controls received the citrate buffer alone. Mice were housed in individual cages and pair-fed for 8 days (19). In other studies, C57BL/ 6 mice were anesthetized and underwent bilateral $\operatorname{ADX}(1,3)$. ADX mice were given $0.45 \% \mathrm{NaCl}$ and allowed to recover for at least 7 days before being divided into 4 groups: (a) ADX mice treated with STZ (AS); (b) ADX mice treated with STZ and $20 \mu \mathrm{g} / \mathrm{kg} / \mathrm{d}$ Dex (ASD); (c) ADX mice treated with the same dose of Dex (AD); and (d) untreated ADX mice (A). Eight days after STZ, mice were anesthetized, arterial blood was collected to measure blood glucose, and muscles were removed. To study fasting, control lox/lox and MIRKO or MGRKO mice at 8 weeks of age were housed in individual cages and given free access to water. Food was withdrawn for 36 hours, and at sacrifice, aortic blood was obtained to measure corticosterone level (R\&D Laboratories). In other experiments, we measured corticosterone production by collecting urine over 24 hours in mice housed in individual cages (1).

PI3K activity assay. Gastrocnemius muscles $(\sim 100 \mathrm{mg})$ were homogenized for 5 minutes in $1 \mathrm{ml}$ cold buffer A $(137 \mathrm{mM} \mathrm{NaCl}, 20 \mathrm{mM}$ Tris- $\mathrm{HCl}$ [pH 7.4]; $1 \mathrm{mM} \mathrm{CaCl} 2 ; 1 \mathrm{mM} \mathrm{MgCl}_{2} ; 0.1 \mathrm{mM} \mathrm{Na}_{3} \mathrm{VO}_{4}, 2 \mu \mathrm{g} / \mathrm{ml}$ of both leupeptin and aprotinin, $1 \mathrm{mM} \mathrm{PMSF}$ and $1 \%$ Nonidet-P40). The mixture was kept on ice for 60 minutes before centrifugation $(16,000 \mathrm{~g}$ for 10 minutes). The supernatant ( $2 \mathrm{mg}$ muscle proteins) was immunoprecipitated with $4 \mu \mathrm{g}$ anti-IRS- 1 or anti-GR antibody overnight at $4{ }^{\circ} \mathrm{C}$. Protein A/G $(25 \mu \mathrm{l})$ agarose beads (Santa Cruz Biotechnology Inc.) were added and incubated for 2 hours at $4{ }^{\circ} \mathrm{C}$. The immunoprecipitates were washed 3 times with buffer $\mathrm{A}$ and 3 times with TNE buffer ( $20 \mathrm{mM}$ Tris- $\mathrm{HCl}$ [pH 7.4], $100 \mathrm{mM}$ $\mathrm{NaCl}$, and $0.5 \mathrm{mM}$ EGTA) before suspension in $50 \mu \mathrm{l}$ of PI3K reaction buffer containing $15 \mu \mathrm{g}$ of phosphatidylinositol (Sigma-Aldrich). Reactions were performed as described previously $(6,40)$.

Northern and Western blotting. Total RNA was isolated from the gastrocnemius muscles using Tri Reagent (Sigma-Aldrich), and Northern blot analysis was performed as described previously $(6,40)$. For Western blots, gastrocnemius muscle $(\sim 100 \mathrm{mg})$ was homogenized for 5 minutes in $1 \mathrm{ml}$ cold RIPA buffer (50 mM Tris [pH 7.4]; 1\% NP-40; 0.25\% Na deoxycholate; 150 $\mathrm{mM} \mathrm{NaCl} ; 1 \mathrm{mM}$ EDTA; $1 \mathrm{mM} \mathrm{PMSF} ; 1 \mathrm{mM} \mathrm{Na}_{3} \mathrm{VO}_{4} ; 1 \mathrm{mM} \mathrm{NaF}$ and $2 \mu \mathrm{g} /$ $\mathrm{ml}$ each of aprotinin, leupeptin, and pepstatin). The mixture was incubated on ice for 60 minutes. Homogenates were centrifuged $(14,000 \mathrm{~g})$ at $4{ }^{\circ} \mathrm{C}$ for 10 minutes, and the supernatants were assayed using immunoprecipitation and antibodies. Proteins were separated by electrophoresis on 10\% SDSpolyacrylamide gels and blotted on PVDF membranes. After blocking with $5 \%$ dry milk $/ 0.1 \%$ Tween- 20 , primary antibodies were added, and they were detected using anti-rabbit or anti-mouse IgG conjugated with IRDye 700 or IRDye 800 (LI-COR) and analyzed as described previously (40).

Myofiber size. Sections $(5 \mu \mathrm{m})$ of TA muscles were treated with DAKO blocking solution before incubation with primary antibodies for 16 hours at $4{ }^{\circ} \mathrm{C}$. After washing, slides were incubated for 30 minutes at room temperature with Alexa Fluor 488 or 560 secondary antibodies (1:800; Invitrogen). Muscle myofiber sizes were determined by Image-Pro software (40). Approximately 300 myofibers from TA muscles of each mouse were measured, and the pattern was confirmed in at least 3 mice with each condition.

Cell culture and transfection. The PECFP-GR-CFP vector was provided by M. Nishi (Kyoto Prefectural University of Medicine, Kyoto, Japan). The pEXEF1-p85-YFP vector was generated by fusion of mouse p $85 \alpha \mathrm{cDNA}$ and YFP. The GR-CFP plasmid $(0.1 \mu \mathrm{g} /$ dish $)$ was transiently transfected into COS1 cells with or without cotransfection of p85-YFP $(0.1 \mu \mathrm{g} /$ dish $)$ using Lipofectamine (Invitrogen). Transfected cells were cultured in Delta $\mathrm{T}$ dishes (Fisher) in DMEM containing 10\% charcoal-stripped FBS; 16 hours after transfection, cells were incubated in serum-free medium for 5 hours. Cells were then treated with $50 \mathrm{ng} / \mathrm{ml}$ insulin and/or $100 \mathrm{nM} \mathrm{Dex}$ (Sigma-Aldrich). Nuclear and cytosolic proteins were separated as described previously (41).

FRET analysis. To image the glucocorticoid-induced interaction between the GR and p85, COS1 cells cotransfected with GR-CFP and p85-YFP 
were kept at $37^{\circ} \mathrm{C}$ in a thermostatic chamber and examined with a Zeiss LSM510 confocal microscope. Cells exhibiting nearly the same fluorescence intensity as the GR-CFP (donor) and the p85-YFP (acceptor) were selected and examined. CFP was excited with a 458-nm laser, and its emission was sampled between 480 and $520 \mathrm{~nm}$. Emission-sensitized YFP fluorescence was collected between 526 and $558 \mathrm{~nm}$ (42). CFP and YFP emissions were acquired simultaneously with a Zeiss Plan-Apochromat $63 \times / 1.4$ oil objective. After subtracting the background measured in areas without cells, the sensitized YFP emission was used to calculate the ratio of YFP/ CFP pixel by pixel using Metamorph software (Molecular Devices). Cells were treated with $100 \mathrm{nM}$ Dex, GR-CFP was excited, and the emission-sensitized p85-YFP fluorescence was measured over 60 minutes. Because the levels of protein expression of the donor, GR-CFP, and the acceptor, p85YFP, molecules in each cell were not exactly the same, we normalized the fluorescence intensity in each cell by dividing the fluorescence intensity after Dex treatment by that measured before treatment (36). The results are representative images obtained from 3 independent experiments.

GR gene mutation and electroporation. The PECFP-GR-CFP plasmid was used to mutate the GR receptor at site 515 using the Quickchange kit (Stratagene) to change Lys to Asn (35). TA muscles of anesthetized MGRKO mice were injected with $50 \mu \mathrm{g}$ DNA in $30 \mu \mathrm{l}$ saline. Electric pulses were applied by 2 stainless steel electrodes on each side of the leg $(100 \mathrm{~V} / \mathrm{cm}, 5$ pulses of $10 \mathrm{~ms}$ at 200-ms intervals) (11). After 7 days, STZ was injected to induce acute diabetes, and the muscles were studied 7 days later; there was no evidence for necrosis or inflammation as a result of this procedure.

In vitro assay to detect competition between IRS-1 and GR for PI3K. C2C12 myotubes were exposed to $5 \mu \mathrm{g} / \mathrm{ml}$ insulin for 10 minutes before being lysed in cold buffer A. Two micrograms anti-IRS-1 antibodies were added to $500 \mu \mathrm{g}$ of cell lysate; anti-rabbit IgG agarose beads were added, and the mixture was incubated for 16 hours. After washing 3 times with cold HEPES buffer (10 mM HEPES, pH 7.4, $120 \mathrm{mM} \mathrm{NaCl}, 2 \mathrm{mM}$ dithiothreitol), the agarose beads were collected and mixed with the HEPES buffer that contained different concentrations of human recombinant GR (MBL) plus $5 \mu$ of an ATP-regenerating system (50 mM ATP, $250 \mathrm{mM}$ creatine phosphate, $20 \mathrm{mM} \mathrm{Mg}$ acetate, and $100 \mathrm{U} / \mathrm{ml}$ creatine phosphokinase). After incubation at $32^{\circ} \mathrm{C}$ for 20 minutes, the beads were washed with TNE buffer. The beads were collected, and either PI3K activity was measured or they were treated with loading buffer before being subjected to Western blotting.

Statistics. Values are presented as mean \pm SEM and results analyzed using 2 -tailed Student's $t$ test when results from 2 experimental groups were compared or using ANOVA when data from 3 groups were studied. For ANOVA analyses, pairwise comparisons were made by the Student-Newman-Keuls test. $P$ values less than 0.05 were considered significant.

\section{Acknowledgments}

We thank Yan Bi for assistance with the FRET analysis. The work was supported by NIH grants R37 DK37175, P50 DK64233, R01 DK62828; Huiling Wang was supported by Shanghai STC grant 07QH14020.

Received for publication February 2, 2009, and accepted in revised form July 22, 2009.

Address correspondence to: William E. Mitch or Jie Du, Nephrology Division, M/S: BCM 285, One Baylor Plaza, Alkek N-520, Houston, Texas 77030, USA. Phone: (713) 798-8350; Fax: (713) 798-5010; E-mail: mitch@bcm.edu (W.E. Mitch). Phone: (713) 7982032; Fax: (713) 798-5010; E-mail: jdu@bcm.edu (J. Du).
1. May, R.C., Kelly, R.A., and Mitch, W.E. 1986. Metabolic acidosis stimulates protein degradation in rat muscle by a glucocorticoid-dependent mechanism. J. Clin. Invest. 77:614-621.

2. Wing, S.S., and Goldberg, A.L. 1993. Glucocorticoids activate the ATP-ubiquitin-dependent proteolytic system in skeletal muscle during fasting. Am.J. Physiol. 264:E668-E676.

3. Mitch, W.E., et al. 1999. Evaluation of signals activating ubiquitin-proteasome proteolysis in a model of muscle wasting. Am. J. Physiol. 276:C1132-C1138.

4. Tiao, G., et al. 1996. Energy-ubiquitin-dependent muscle proteolysis during sepsis in rats is regulated by glucocorticoids. J. Clin. Invest. 97:339-348.

5. Song, Y.-H., et al. 2005. Muscle-specific expression of insulin-like growth factor-1 blocks angiotensin II-induced skeletal muscle wasting. J. Clin. Invest. 115:451-458.

6. Lee, S.W., et al. 2004. Regulation of muscle protein degradation: coordinated control of apoptotic and ubiquitin-proteasome systems by phosphatidylinositol 3 kinase. J. Am. Soc. Nephrol. 15:1537-1545.

7. Du, J., et al. 2004. Activation of caspase 3 is an initial step triggering muscle proteolysis in catabolic conditions. J. Clin. Invest. 113:115-123.

8. Glass, D.J. 2003. Signalling pathways that mediate skeletal muscle hypertrophy and atrophy. Nat. Cell Biol. 5:87-90.

9. Bodine, S.C., et al. 2001. Akt/mTOR pathway is a crucial regulator of skeletal muscle hypertrophy and can prevent muscle atrophy in vivo. Nat. Cell Biol. 3:1014-1019.

10. Wang, X.H., Hu, Z., Hu, J.P., Du, J., and Mitch, W.E. 2006. Insulin resistance accelerates muscle protein degradation: activation of the ubiquitin-proteasome pathway by defects in muscle cell signaling. Endocrinology. 147:4160-4168.

11. Sandri, M., et al. 2004. Foxo transcription fac tors induce the atrophy-related ubiquitin ligase atrogin-1 and cause skeletal muscle atrophy. Cell. 117:399-412.

12. Stitt, T.N., et al. 2004. The IGF-1/PI3K/Akt pathway prevents expression of muscle atrophy-induced ubiquitin ligases by inhibiting FOXO transcription factors. Mol. Cell. 14:395-403.

13. Sacheck, J.M., Ohtsuka, A., McLary, S.C., and Goldberg, A.L. 2004. IGF-1 stimulates muscle growth by suppressing protein breakdown and expression of atrophy-related ubiquitin ligases, atrogin-1 and MuRF1. Am. J. Physiol. Endocrinol. Metab. 287:E591-E601.

14. Lai, K.M., et al. 2004. Conditional activation of Akt in adult skeletal muscle induces rapid hypertrophy. Mol. Cell. Biol. 24:9295-9304.

15. Lecker, S.H., Goldberg, A.L., and Mitch, W.E. 2006. Protein degradation by the ubiquitin-proteasome pathway in normal and disease states. J. Am. Soc. Nephrol. 17:1807-1819.

16. Bailey, J.L., Price, S.R., Zheng, B., Hu, Z., and Mitch, W.E. 2006. Chronic kidney disease causes defects in signaling through the insulin receptor substrate/phosphatidylinositol 3-kinase/Akt pathway: implications for muscle atroply. J. Am. Soc. Nephrol. 17:1388-1394.

17. DeFronzo, R.A., and Beckles, A.D. 1979. Glucose intolerance following chronic metabolic acidosis in man. Am. J. Physiol. 236:E328-E334.

18. Mitch, W.E., et al. 1994. Metabolic acidosis stimulates muscle protein degradation by activating the ATP-dependent pathway involving ubiquitin and proteasomes. J. Clin. Invest. 93:2127-2133.

19. Price, S.R., et al. 1996. Muscle wasting in insulinopenic rats results from activation of the ATPdependent, ubiquitin-proteasome pathway by a mechanism including gene transcription. J. Clin. Invest. 98:1703-1708.

20. Hasselgren, P.-O., Warner, B.W., James, H., Takehara, H., and Fischer, J.E. 1987. Effect of insulin on amino acid uptake and protein turnover in skeletal muscle from septic rats: evidence for insulin resistance of protein degradation. Arch. Surg. 122:228-233.

21. Bruning, J.C., et al. 1998. A muscle -specific insulin receptor knockout exhibits features of the metabolic syndrome of NIDDM without altering glucose tolerance. Mol. Cell. 2:559-569.

22. Kayali, A.G., Young, V.R., and Goodman, M.N. 1987. Sensitivity of myofibrillar proteins to glucocorticoid induced muscle proteolysis. Am. J. Physiol. 252:E621-E626.

23. Sandri, M. 2008. Signaling in muscle atrophy and hypertrophy. Physiology (Bethesda). 23:160-170.

24. Saad, M.J.A., Folli, F., Kahn, J.A., and Kahn, C.R. 1993. Modulation of insulin receptor, insulin receptor substrate-1, and phosphatidylinositol 3-kinase in liver and muscle of dexamethasonetreated rats. J. Clin. Invest. 92:2065-2072.

25. Giorgino, F., and Smith, R.J. 1995. Dexamethasone enhances insulin-like growth factor-I effects on skeletal muscle cell proliferation: role of specific intracellular signaling pathways. J. Clin. Invest. 96:1473-1483

26. Ueki, K., et al. 2002. Molecular balance between the regulatory and catalytic subunits of phosphoinositide 3-kinase regulates cell signaling and survival. Mol. Cell. Biol. 22:965-977.

27. Schakman, O., et al. 2008. Role of Akt/GSK-3beta/ beta-catenin transduction pathway in the muscle anti-atrophy action of insulin-like growth factor-I in glucocorticoid-treated rats. Endocrinology. 149:3900-3908

28. Waddell, D.S., et al. 2008. The glucocorticoid receptor and FOXO1 synergistically activate the skeletal muscle atrophy-associated MuRF1 gene. Am.J. Physiol. Endocrinol. Metab. 295:E785-E797.

29. Limbourg, F.P., et al. 2002. Rapid nontranscriptional activation of endothelial nitric oxide synthase mediates increased cerebral blood flow and 
stroke protection by corticosteroids. J. Clin. Invest. 110:1729-1738.

30. Song, I.H., and Buttgereit, F. 2006. Non-genomic glucocorticoid effects to provide the basis for new drug developments. Mol. Cell. Endocrinol. 246:142-146.

31. Simoncini, R., et al. 2004. Interaction of oestrogen receptor with the regulatory subunit of phophatidylinositol-3-OH kinase. Nature. 407:538-541.

32. De Bosscher, K., et al. 1997. Glucocorticoidmediated repression of nuclear factor-kB-dependent transcription involves direct interference with transactivation. Proc. Natl. Acad. Sci. U. S. A 94:13504-13509.

33. Auphan, N., DiDonato, J.A., Rosette, C., Helmberg, A., and Karin, M. 1995. Role of transcriptional activation of $\mathrm{IkB}$ in mediation of immunosuppression by glucocorticoids. Science. 270:286-290.

34. Helmberg, A., Auphan, N., Caelles, C., and Karin, M.
1995. Glucocorticoid-induced apoptosis of human leukemic cells is caused by the repressive function of the glucocorticoid receptor. EMBOJ. 14:452-460.

35. Tang, Y., Ramakrishnan, C., Thomas, J., and DeFranco, D.B. 1997. A role for HDJ-2/HSDJ in correcting subnuclear trafficking, transactivation, and transrepression defects of a glucocorticoid receptor zinc finger mutant. Mol. Biol. Cell. 8:795-809.

36. Nishi, M., Tanaka, M., Matsuda, K., Sunaguchi, M., and Kawata, M. 2004. Visualization of glucocorticoid receptor and mineralocorticoid receptor interactions in living cells with GFP-based fluorescence resonance energy transfer. J. Neurosci. 24:4918-4927.

37. Luo, J., Field, S.J., Lee, J.Y., Engelman, J.A., and Cantley, L.C. 2005. The p85 regulatory subunit of phosphoinositide 3-kinase down-regulates IRS-1 signaling via the formation of a sequestration com- plex. J. Cell Biol. 170:455-464.

38. Bruna, A., Nicolas, M., Munoz, A., Kyriakis, J.M., and Caelles, C. 2003. Glucocorticoid receptor-JNK interaction mediates inhibition of the JNK pathway by glucocorticoids. EMBO J. 22:6035-6044.

39. Slusher, M.A., and Roberts, S. 1957. Fate of adrenal ascorbic acid: relationship to corticosteroid secretion. Endocrinology. 61:98-105.

40. Hu, Z., et al. 2007. PTEN expression contributes to the regulation of muscle protein degradation in diabetes. Diabetes. 56:2449-2456.

41. Zhang, L., et al. 2005. Dual pathways for nuclear factor kappaB activation by angiotensin II in vascular smooth muscle: phosphorylation of $\mathrm{p} 65$ by IkappaB kinase and ribosomal kinase. Circ. Res. 97:975-982.

42. Piston, D.W., and Kremers, G.-J. 2007. Fluorescent protein FRET: the good, the bad and the ugly. Trends Biochem. Sci. 32:407-414. 